\title{
Determination of the Lipophilicity of Some New Derivatives of Semi- carbazide and 1,2,4-Triazol-5-one with Potential Antibacterial Activity
}

\author{
Monika Pitucha $^{\mathrm{a}}$, Beata Polak ${ }^{\mathrm{b}}$, Ryszard Świeboda $^{\mathrm{c}}$, Urszula Kosikowska ${ }^{\mathrm{d}}$, \\ and Anna Malm ${ }^{\text {d }}$ \\ ${ }^{a}$ Department of Organic Chemistry, Medical University, Staszica 6, 20-081 Lublin, Poland \\ ${ }^{b}$ Department of Physical Chemistry, Medical University, Staszica 6, 20-081 Lublin, Poland \\ c Department of Inorganic Chemistry, Chair of Chemistry, Medical University, Staszica 6, \\ 20-081 Lublin, Poland \\ d Department of Pharmaceutical Microbiology, Medical University, Chodzki 1, 20-093 Lublin, \\ Poland
}

Reprint requests to Dr. Monika Pitucha. E-mail: monika.pitucha@am.lublin.pl

Z. Naturforsch. 2009, 64b, 570-576; received February 15, 2009

\begin{abstract}
The chromatographic behavior of new derivatives of 1,2,4-triazol-5-one and semicarbazide was determined. The lipophilicity was confirmed by the use of a RP-TLC method. The partition coefficients were calculated by use of theoretical procedures. The correlation between theoretical and experimental lipophilicity was determined. All obtained compounds were tested for their antimicrobial activity.
\end{abstract}

Key words: Semicarbazide, 1,2,4-Triazol-5-one, Lipophilicity, Antibacterial Activity

\section{Introduction}

Many people around the world suffer from microbial infections, including opportunistic or recurrent diseases associated usually with immunosuppressants. In addition, the effectiveness of available antibacterial agents is diminishing due to developing, increasing and spreading of microbial resistance [1,2]. Therefore, synthesis of novel compounds of potential antimicrobial activity seems to be necessary. A great number of 1,2,4-triazoles and 4,5-dihydro- $1 \mathrm{H}$-1,2,4-triazol-5ones have a wide range of biological activities such as analgesic [3], antibacterial [4-7], fungicidal [8,9], anti-inflammatory [10], antiviral [11,12], anticonvulsant [13], and antitumor $[14,15]$ properties. Due to these properties, the triazole moiety is a structural element of drugs such as fluconazole, itraconazole, vorozole, letrozole, and anastrozole [16-18].

It is known that the biological activity of the molecules is related to their structure and physicochemical properties. The lipophilicity denotes the physical property of a molecule which influences the transport of compounds through the biological system. This property of molecules could be used to characterize bioavailability, distribution, activity, toxicity, and other processes $[19,20]$. Lipophilicity is usually measured by the partition coefficient of the investigated compound between a nonpolar, organic phase and water. It can be determined by the traditional "shake-flask" partition method between $n$-octanol (or other organic compounds, immiscible with water) and water. Similar conditions of determination can be achieved by the use of reversed phase chromatography (both TLC and HPLC methods) [21-23]. A linear relationship between the retention parameters and the concentration $(\Phi)$ of an organic modifier in the aqueous mobile phase has to be established for a successful chromatographic measurement of lipophilicity [24-27]. The lipophilicity of some biologically active compounds was experimentally determined [28-32]. Some authors determined the lipophilicity of a solute as the $\log P_{\mathrm{TLC}}$ coefficient $\left(\log P_{\mathrm{TLC}}=\right.$ experimental lipophilicity obtained for standards solutes in investigation condition). For this purpose the $R_{\mathrm{MW}}\left(R_{\mathrm{MW}}=\right.$ intercept in equation $R_{\mathrm{M}}=R_{\mathrm{MW}}+\mathrm{a} C$; relative lipophilicity) values of investigated compounds and standard solutes were compared [33-35]. The evaluation of the lipophilicity of the solute with potential activity allows the estimation of its biological activity in compliance with the Hansch theory [36], and pertinent research refers to Quantitative Structure Activity Relationships (QSAR) [37-40].

The objective of the studies reported here is a chromatographic analysis of two series of newly synthe- 
<smiles></smiles>

$\mathrm{R}=4-\mathrm{BrC}_{6} \mathrm{H}_{4}(\mathbf{a}), \mathrm{CH}_{2} \mathrm{C}_{6} \mathrm{H}_{5}(\mathbf{b}), 4-\mathrm{C}_{2} \mathrm{H}_{5} \mathrm{OC}_{6} \mathrm{H}_{4}(\mathbf{c}), \mathrm{C}_{6} \mathrm{H}_{11}(\mathbf{d}), \mathrm{C}_{6} \mathrm{H}_{5}(\mathbf{e}), \mathrm{C}_{2} \mathrm{H}_{5}(\mathbf{f})$
Scheme 1. sized derivatives of 1,2,4-triazol-5-one and semicarbazide with potential antibacterial activity.

\section{Results and Discussion}

The new 3-(1-methyl-pyrrol-2-yl)-4-substituted 4,5dihydro-1H-1,2,4-triazol-5-one derivatives $\mathbf{3 a}-\mathbf{f}$ were synthesized by cyclization of semicarbazide derivatives $\mathbf{2 a}-\mathbf{f}$ in alkaline medium (Scheme 1). They

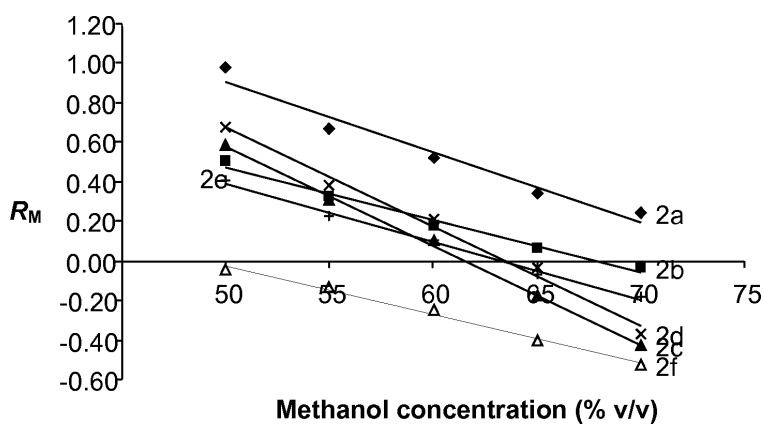

Fig. 1. Relationships between $R_{\mathrm{M}}$ of semicarbazide derivatives and concentration of methanol in the mobile phase.

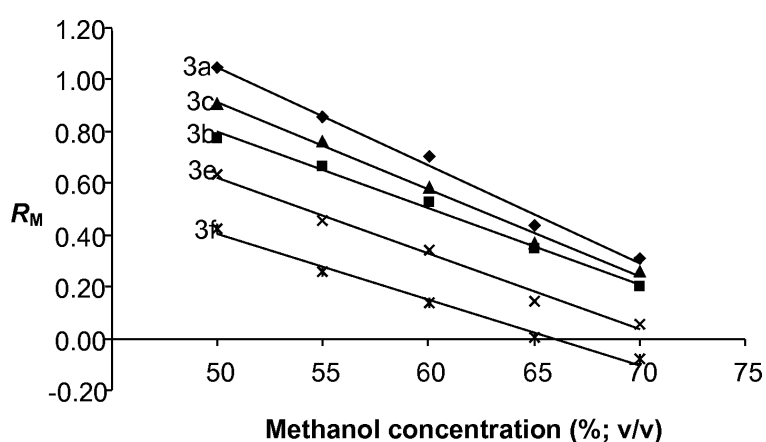

Fig. 2. Relationships between $R_{\mathrm{M}}$ of 1,2,4-triazol-5-one derivatives and concentration of methanol in the mobile phase.

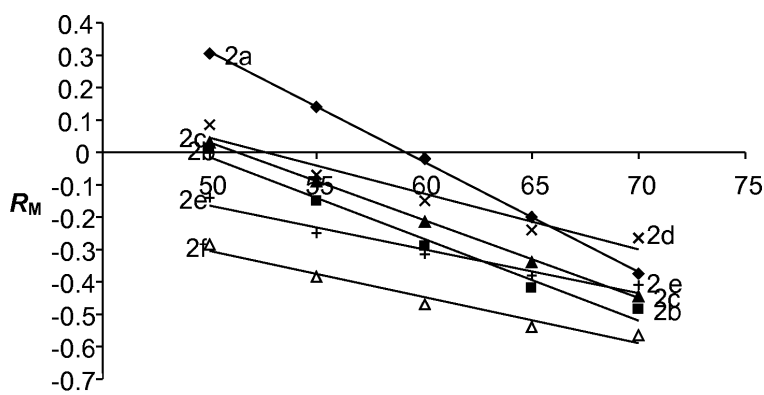

ACN concentration (\% v/v)

Fig. 3. Relationships between $R_{\mathrm{M}}$ of semicarbazide derivatives and concentration of acetonitrile in the mobile phase.

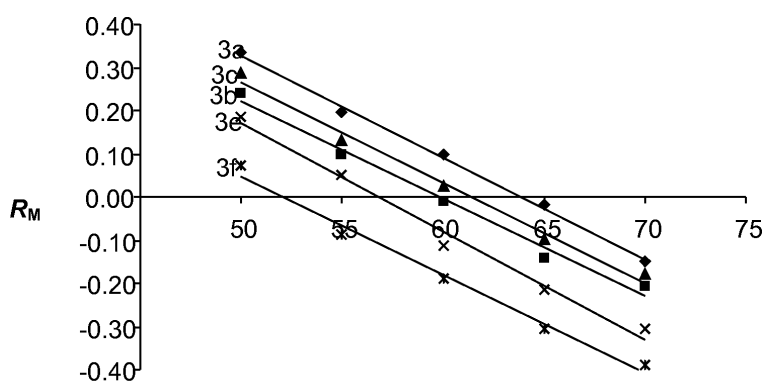

ACN concentration $(\% \mathrm{v} / \mathrm{v})$

Fig. 4. Relationships between $R_{\mathrm{M}}$ of 1,2,4-triazol-5-one derivatives and concentration of acetonitrile in the mobile phase.

were then characterized by elemental analyses, IR, ${ }^{1} \mathrm{H}$ and ${ }^{13} \mathrm{C}$ NMR spectroscopy, as well as by MS. The lipophilicity of selected solutes $(\mathbf{2} \mathbf{a}-\mathbf{f}, \mathbf{3 a}-\mathbf{c}$, $\mathbf{3 e}-\mathbf{f})$ was chromatographically determined. Experimental data obtained by the RP-TLC method are presented as plots of $R_{\mathrm{M}}\left(R_{\mathrm{M}}=\log \frac{\left(1-R_{\mathrm{F}}\right)}{R_{\mathrm{F}}}\right)$ values $v$ s. concentration of methanol or acetonitrile $(\% \mathrm{v} / \mathrm{v})$ in the mobile phase (Figs. 1-4). Linear relationships showed the regular dependence of retention on the organic 
modifier concentration. A similar range of $R_{\mathrm{M}}$ values was determined for both kinds of solutes. For both kinds of mobile phase polar modifiers, the sequence of $R_{\mathrm{M}}$ values for semicarbazide derivatives was (from the biggest to the smallest values) as follows: $\mathbf{2 a}, \mathbf{2} \mathbf{b}$, 2e, 2d, 2c, 2f; for 1,2,4-triazol-5-one derivatives it was as follows: $\mathbf{3 a}, \mathbf{3 c}, \mathbf{3 b}, \mathbf{3 e}$ and $\mathbf{3 f}$. The sequence of solutes did not depend on the kind of the polar modifier. However, the results indicate that for solutes of the semicarbazide series of investigated compounds, the sequence changes when the concentration of the polar modifier is increased. It was also noticed that the highest $R_{\mathrm{M}}$ values were found when methanol was utilized as polar modifier. The sequence of solutes depended on the kind of substituent. The smallest adsorption (the smallest $R_{\mathrm{M}}$ values) in both groups of solutes was determined for compounds with alkyl substituents, the biggest adsorption was measured for compounds with a bromophenyl group. Relationships of $R_{\mathrm{M}} v s$. organic modifier concentration $(C)$ allowed to determine the $R_{\mathrm{MW}}$ and $\Phi$ values according to the SoczewinskiWachtmeister equation [41]:

$$
R_{\mathrm{M}}=R_{\mathrm{MW}}+\mathrm{b} C
$$

where $C$ is the percentage of methanol in the mobile phase and $\Phi$ is the ratio $R_{\mathrm{MW}} / \mathrm{b}$.

Both coefficients could be used for lipophilicity determination. The values of coefficients as well as the other parameters of the linear correlation of $R_{\mathrm{M}}$ $v s$. organic modifier concentration are presented in Table 1. $R_{\mathrm{MW}}$ values are in the range from 0.409 to 3.186 for semicarbazide derivatives (solutes for the semicarbazide group) and from 1.188 to 2.943 for 1,2,4-triazol-5-ones. Among the semicarbazide derivatives, the smallest values were determined for solutes with an ethyl substituent (2f), and the highest value was found for a solute with a cyclohexyl substituent (2d, methanol as modifier). For 1,2,4-triazol5-one derivatives, the smallest $R_{\mathrm{MW}}$ value was obtained for alkyl-substituted solutes, the highest values for bromophenyl-substituted ones. The biggest values of coefficients was reached when methanol was used as mobile phase organic modifier. The second parameter $\Phi$ was very low for both groups of solutes. For semicarbazide derivatives it was in the range $0.013-0.035$, for 1,2,4-triazol-5-one derivatives in the range $0.013-$ 0.019 . Theoretically determined lipophilicity parameters $(\log P)$ of the investigated solutes are given in Table 2 . The $R_{\mathrm{MW}}$ and $\Phi$ coefficients were compared
Table 1. Parameters of the $R_{\mathrm{M}}=\mathrm{a}+\mathrm{bC}$, where $C$ is the concentration of modifier $(\% ; \mathrm{v} / \mathrm{v})$ in the mobile phase. $R^{2}=$ correlation coefficient square of the equation.

\begin{tabular}{|c|c|c|c|c|c|}
\hline & Slope & Intercept & $R^{2}$ & $R_{\mathrm{MW}}$ & $-\Phi$ \\
\hline \multicolumn{6}{|c|}{ Methanol: } \\
\hline $2 \mathbf{a}$ & -0.04 & 2.70 & 0.96 & 2.70 & 0.01 \\
\hline $2 b$ & -0.03 & 1.82 & 0.98 & 1.82 & 0.01 \\
\hline $2 c$ & -0.05 & 3.09 & 0.99 & 3.10 & 0.02 \\
\hline 2d & -0.05 & 3.19 & 0.99 & 3.19 & 0.02 \\
\hline $2 e$ & -0.03 & 1.87 & 0.99 & 1.87 & 0.02 \\
\hline $2 f$ & -0.02 & 1.21 & 0.99 & 1.21 & 0.02 \\
\hline 3a & -0.04 & 2.94 & 0.99 & 2.94 & 0.01 \\
\hline 3b & -0.03 & 2.26 & 0.99 & 2.26 & 0.01 \\
\hline $3 c$ & -0.03 & 2.60 & 0.99 & 2.60 & 0.01 \\
\hline $3 e$ & -0.03 & 2.09 & 0.99 & 2.09 & 0.01 \\
\hline $3 f$ & -0.02 & 1.66 & 0.99 & 1.66 & 0.01 \\
\hline \multicolumn{6}{|c|}{ Acetonitrile: } \\
\hline $2 \mathbf{a}$ & -0.03 & 2.01 & 0.99 & 2.1 & 0.02 \\
\hline $2 b$ & -0.02 & 1.25 & 0.98 & 1.25 & 0.02 \\
\hline $2 \mathrm{c}$ & -0.02 & 1.22 & 0.99 & 1.22 & 0.02 \\
\hline $2 d$ & -0.02 & 0.92 & 0.94 & 0.91 & 0.02 \\
\hline $2 e$ & -0.01 & 0.51 & 0.96 & 0.51 & 0.03 \\
\hline $2 \mathrm{f}$ & -0.01 & 0.41 & 0.96 & 0.41 & 0.03 \\
\hline 3a & -0.02 & 1.51 & 0.99 & 1.51 & 0.02 \\
\hline 3b & -0.02 & 1.35 & 0.98 & 1.35 & 0.02 \\
\hline $3 c$ & -0.02 & 1.42 & 0.98 & 1.42 & 0.02 \\
\hline $3 e$ & -0.02 & 1.42 & 0.99 & 1.42 & 0.02 \\
\hline 3f & -0.02 & 1.19 & 0.99 & 1.19 & 0.02 \\
\hline
\end{tabular}

with theoretically calculated lipophilicity coefficients. The results of this comparison for each group of investigated solutes are presented in Table 3 . The best correlations for the 1,2,4-triazole group of solutes measured as Pearson coefficient was achieved for $\log P_{\text {KOWIN }}$ and for $\Phi_{\mathrm{ACN}}(0.9973)$ and for Pallas ANNLOG P (0.9916) and for $R_{\mathrm{MWMeOH}}$. The correlations for the semicarbazide group of solutes was poorer. However the best results were achieved for $\mathrm{MMP} \mathrm{Q} \log P$ and $\Phi_{\mathrm{ACN}}$.

The in vitro antimicrobial activities of compounds $\mathbf{2 a}-\mathbf{f}$ and 3a-f were evaluated using the agar-well diffusion method (at concentrations $1000-5000 \mathrm{mg} \mathrm{L}^{-1}$ per well). Our results showed that all compounds were inactive against four reference strains of fungi. Similar results were obtained for two reference species of Staphylococcus spp. It was found that the tested compounds possess some antibacterial activity against the reference strains of Gram-positive or Gram-negative bacteria as revealed by a complete or partial reduction $(20-80 \%)$ of the growth around the wells with or without the clear zone, depending on the strains and the concentration of the compound (1000-5000 mg $\mathrm{L}^{-1}$ per well); the zone diameter of growth inhibition was $\geq 12-15 \mathrm{~mm}$. The antibacterial effect of 
Table 2. Theoretically determined $\log P$ coefficients of investigated solutes.

\begin{tabular}{|c|c|c|c|c|c|c|c|c|c|c|c|}
\hline & $2 \mathbf{a}$ & $2 \mathbf{b}$ & $2 c$ & $2 d$ & $2 \mathrm{e}$ & $2 f$ & $\mathbf{3 a}$ & $3 \mathbf{b}$ & $3 c$ & $3 \mathbf{e}$ & $3 f$ \\
\hline Molinspiration & 2.36 & 1.55 & 1.98 & 1.76 & 1.55 & 0.73 & 2.47 & 1.97 & 2.10 & 1.67 & 0.77 \\
\hline ALOGPS & 1.98 & 1.40 & 1.77 & 1.09 & 1.40 & 0.43 & 3.20 & 2.48 & 2.99 & 2.43 & 1.52 \\
\hline $\log P_{\text {KOWIN }}$ & 2.32 & 1.43 & 1.10 & 1.90 & 1.43 & 0.61 & 3.45 & 2.86 & 3.14 & 2.56 & 1.64 \\
\hline Pallas ANNLOG $P$ & 1.61 & 0.60 & 1.20 & 2.01 & 0.64 & 0.18 & 2.32 & 1.52 & 1.92 & 1.29 & 0.49 \\
\hline Pallas ATOMIC6 & 2.58 & 1.63 & 1.98 & 2.01 & 1.68 & 0.96 & 3.14 & 2.13 & 2.53 & 2.24 & 0.95 \\
\hline Pallas CDI-REKKER & 1.14 & 0.02 & 0.79 & 0.60 & 0.19 & -0.60 & 2.15 & 1.24 & 1.79 & 1.20 & 0.10 \\
\hline Pallas COMBINED & 1.74 & 0.74 & 1.31 & 2.01 & 0.78 & 0.28 & 2.43 & 1.60 & 2.00 & 1.42 & 0.56 \\
\hline CHEM3D $\log P$ & 1.53 & 0.70 & 0.91 & 0.58 & 0.70 & -0.14 & 2.65 & 1.89 & 2.04 & 1.83 & 0.50 \\
\hline $\begin{array}{l}\text { CHEM3D Partition Coefficient } \\
\text { (Octanol/Water) }\end{array}$ & 2.23 & 1.09 & 1.71 & 1.41 & 1.09 & 0.44 & 3.53 & 2.67 & 2.88 & 2.67 & 1.22 \\
\hline SciLog $P$ & 5.88 & 5.53 & 5.85 & 5.64 & 5.51 & 5.24 & 5.75 & 5.50 & 5.73 & 5.33 & 4.80 \\
\hline MMP Q $\log P$ & -1.07 & -1.69 & -1.37 & -1.33 & -1.88 & -2.46 & 2.73 & 2.59 & 2.63 & 2.11 & 1.01 \\
\hline MMP log oct/wat & 6.11 & 5.59 & 5.71 & 5.47 & 5.69 & 5.05 & 5.60 & 5.33 & 5.08 & 5.08 & 4.40 \\
\hline TITAN $\log P$ & 0.87 & 0.11 & 0.26 & -0.07 & 0.05 & -0.79 & 2.00 & 1.24 & 1.38 & 1.17 & -0.15 \\
\hline HYPERCHEM & 1.90 & 1.11 & 1.20 & 1.01 & 1.11 & 0.24 & 2.55 & 1.85 & 1.85 & 1.76 & 0.42 \\
\hline ACD $/ \log P$ & 2.18 & 1.12 & 1.53 & 1.36 & 1.05 & 0.36 & 1.75 & 1.13 & 0.36 & 0.74 & -0.48 \\
\hline
\end{tabular}

the tested compounds showing some activity against various species of bacteria assessed by the agar-well diffusion method was further determined spectrophotometrically by the broth dilution method (31.25 to $\left.500 \mathrm{mg} \mathrm{L}^{-1}\right)$. According to our results, the most effective antibacterial compounds were compound $\mathbf{2 a}$ $\left(\mathrm{MIC}=500 \mathrm{mg} \mathrm{L}^{-1}\right.$ for $B$. cereus ATCC 10876 and P. mirabilis ATCC 12453 and about $84 \%$ inhibition of $M$. luteus ATCC 10240 growth at the same concentration), compound $3 \mathrm{c}$ (MIC $=500 \mathrm{mg} \mathrm{L}^{-1}$ and about $40-$ $50 \%$ inhibition of the growth of E. coli ATCC 25922 in lower concentrations of this compound and by about $60-65 \%$ inhibition of the growth of $K$. pneumoniae ATCC 13883 in concentrations $31.25-500 \mathrm{mg} \mathrm{L}^{-1}$ ) and compound 3d (MIC $=500 \mathrm{mg} \mathrm{L}^{-1}$ for $P$. aeruginosa ATCC 9027 and about $70-60 \%$ inhibition of the growth of K. pneumoniae ATCC 13883 at values ranging from $31.25-500 \mathrm{mg} \mathrm{L}^{-1}$ ).

\section{Conclusion}

The lipophilicity of newly obtained derivatives of 1,2,4-triazol-5-one and semicarbazide was determined. The relationship $R_{\mathrm{M}} v s$. $C$ of a mobile phase polar modifier (methanol or acetonitrile) is linear and allowed to calculate the lipophilicity coefficients. The smallest values of lipophilicity were determined for compounds with alkyl substituents in both groups of solutes. Good correlation between theoretical and experimental lipophilicity was achieved for the 1,2,4-triazol5 -one series of solutes. The results obtained in the study should be of value to further detailed studies on the biological activity of all compounds.

\section{Experimental Section}

Synthesis

All chemicals were purchased from Merck Co. or Lancaster (Gdańsk, Poland) and used without further purification. Melting points were determined in a Fisher-Johns block and are presented withought correction. IR spectra were recorded in $\mathrm{KBr}$ on a Perkin-Elmer 1725X FTIR spectrometer. ${ }^{1} \mathrm{H}$ and ${ }^{13} \mathrm{C}$ NMR spectra were recorded on a Brucer Avance 300 instrument in $\left[\mathrm{D}_{6}\right] \mathrm{DMSO}$ with TMS as internal standard. The mass spectra were obtained on a Finnigan Trace DSQ spectrometer operating at $70 \mathrm{eV}$.

(1-Methyl-pyrrol-2-yl)acetic acid hydrazide (1) was prepared from the reaction of the corresponding carboxylic acid ester with hydrazine hydrate by the method described earlier [42].

Preparation of 1-[(1-methyl-pyrrol-2-yl)acetyl]-4-sub-
stituted semicarbazides $2 \boldsymbol{a}-\boldsymbol{c}$

A mixture of (1-methyl-pyrrol-2-yl)acetic acid hydrazide $1(1.53 \mathrm{~g}, 10 \mathrm{mmol})$ and the appropriate isocyanate (10 mmol) in $10 \mathrm{~mL}$ of $N, N$-dimethylacetamide was kept at r. t. for $24 \mathrm{~h}$, and then water $(40 \mathrm{~mL})$ was added. The precipitate was filtered off and crystallized from ethanol. The physical constants of the products are given below.

Compound 2a: M. p. $200-201{ }^{\circ} \mathrm{C}$. - Yield $86 \%$. - IR (KBr): $v=3287,3037,2960,1490,1696 \mathrm{~cm}^{-1} .-{ }^{1} \mathrm{H}$ NMR (300 MHz, [D 6 ]DMSO): $\delta=3.49\left(\mathrm{~s}, 2 \mathrm{H}, \mathrm{CH}_{2}\right), 3.54(\mathrm{~s}$, $\left.3 \mathrm{H}, \mathrm{NCH}_{3}\right), 5.96-6.63(\mathrm{~m}, 3 \mathrm{H}, \mathrm{Ar}-\mathrm{H}), 7.39-7.45(\mathrm{~m}, 4 \mathrm{H}$, Ar-H), 8.13, 8.87, $9.79(3 \times \mathrm{s}, 3 \mathrm{H}, 3 \mathrm{NH}) .-\mathrm{C}_{14} \mathrm{H}_{15} \mathrm{~N}_{4} \mathrm{O}_{2} \mathrm{Br}$ (351.2): calcd. C 47.87, H 3.30, N 15.95; found C 47.83, H 4.25, N 15.98 .

Compound 2 b: M. p. $150-151{ }^{\circ} \mathrm{C}$. - Yield $76 \%$. - IR (KBr): $v=3251,3029,2974,1494,1682 \mathrm{~cm}^{-1} .-{ }^{1} \mathrm{H}$ NMR (300 MHz, [D 6 ]DMSO): $\delta=3.31\left(\mathrm{~s}, 2 \mathrm{H} \mathrm{CH}_{2}\right.$ ), 3.43 (s, $3 \mathrm{H}$, 


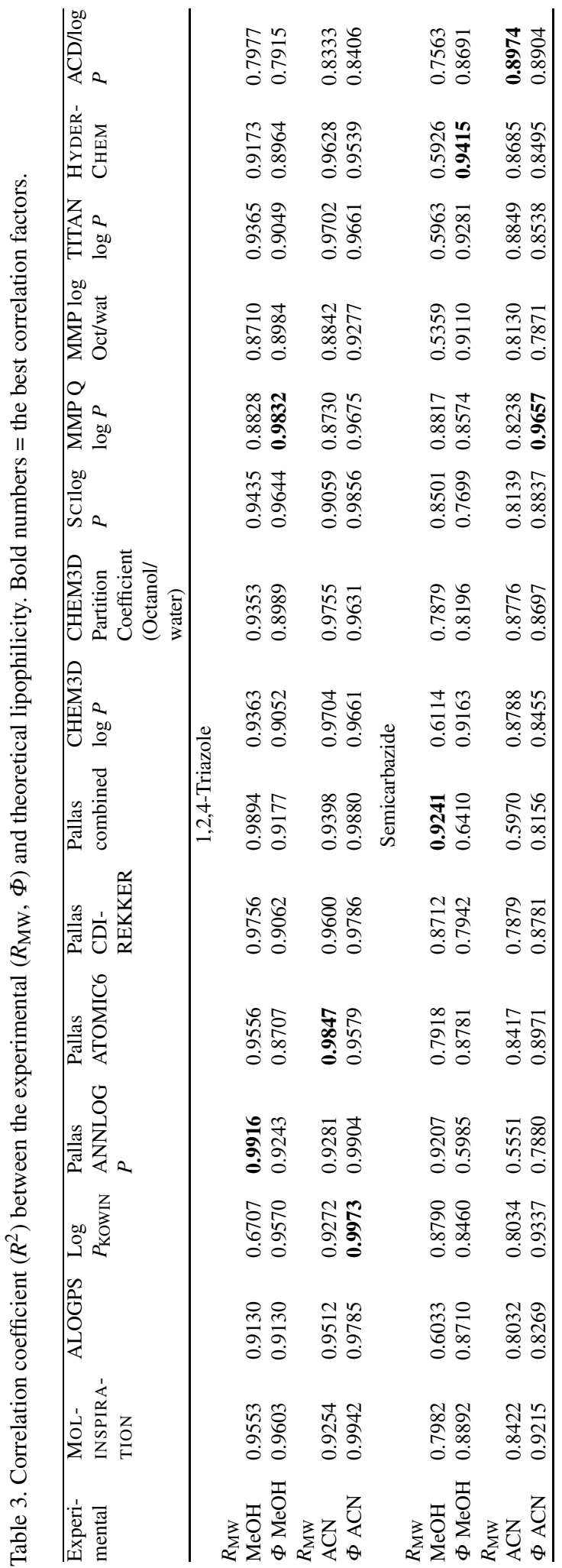

$\left.\mathrm{NCH}_{3}\right), 4.26\left(\mathrm{~s}, 2 \mathrm{H}, \mathrm{CH}_{2}\right), 5.77-6.62(\mathrm{~m}, 3 \mathrm{H}, \mathrm{Ar}-\mathrm{H}), 7.14$ $7.34(\mathrm{~m}, 5 \mathrm{H}, \mathrm{Ar}-\mathrm{H}), 7.86,8.80,9.64(3 \times \mathrm{s}, 3 \mathrm{H}, 3 \mathrm{NH})$. $\mathrm{C}_{15} \mathrm{H}_{18} \mathrm{~N}_{4} \mathrm{O}_{2}$ (286.3): calcd. C 62.92, H 6.33, N 19.56; found C 62.88, H 6.39, N 19.49 .

Compound 2c: M.p. $192-193{ }^{\circ}$ C. - Yield $82 \%$. - IR $(\mathrm{KBr}): v=3283,3036,2974,1494,1682 \mathrm{~cm}^{-1} \cdot-{ }^{1} \mathrm{H}$ NMR (300 MHz, [D 6 DMSO): $\delta=1.29\left(\mathrm{t}, J=7.0 \mathrm{~Hz}, 3 \mathrm{H}, \mathrm{CH}_{3}\right)$, $3.47\left(\mathrm{~s}, 2 \mathrm{H}, \mathrm{CH}_{2}\right), 3.54\left(\mathrm{~s}, 3 \mathrm{H}, \mathrm{NCH}_{3}\right), 3.96(\mathrm{q}, J=6.9 \mathrm{~Hz}$, $\left.2 \mathrm{H}, \mathrm{CH}_{2}\right), 5.81-6.62(\mathrm{~m}, 3 \mathrm{H}, \mathrm{Ar}-\mathrm{H}), 7.14-7.37(\mathrm{~m}, 4 \mathrm{H}$, Ar- $\mathrm{H}), 7.94,8.71,9.74(3 \times \mathrm{s}, 3 \mathrm{H}, 3 \mathrm{NH}) .-\mathrm{C}_{16} \mathrm{H}_{20} \mathrm{~N}_{4} \mathrm{O}_{3}$ (316.3): calcd. C 60.74, H 6.37, N 17.71; found C 60.68, H 6.41, N 17.67. Preparation of 1-[(1-methyl-pyrrol-2-yl)acetyl]-4-sub-
stituted semicarbazides $2 \boldsymbol{d}-\boldsymbol{f}$

(1-Methyl-pyrrol-2-yl)acetic acid hydrazide 1 (1.53 g, $10 \mathrm{mmol}$ ) and the appropriate isocyanate $(10 \mathrm{mmol})$ in $10 \mathrm{~mL}$ of anhydrous diethyl ether were kept at r. t. for $24 \mathrm{~h}$. Then the formed compound was filtered off, washed with diethyl ether and crystallized from ethanol. The physical constants of the compounds prepared are given below.

Compound 2c: M. p. $178-179{ }^{\circ} \mathrm{C}$. - Yield $81 \%$. - IR (KBr): $v=3321,3014,2930,1495,1682 \mathrm{~cm}^{-1} .-{ }^{1} \mathrm{H}$ NMR (300 MHz, [D 6 DMSO): $\delta=1.05-1.72\left(\mathrm{~m}, 10 \mathrm{H}, 5 \mathrm{CH}_{2}\right)$, $3.41\left(\mathrm{~s}, 2 \mathrm{H}, \mathrm{CH}_{2}\right), 3.53\left(\mathrm{~s}, 3 \mathrm{H}, \mathrm{NCH}_{3}\right), 3.61-3.69$ (m, $1 \mathrm{H}, \mathrm{CH}) 5.83-6.61(\mathrm{~m}, 3 \mathrm{H}, \mathrm{Ar}-\mathrm{H}), 7.61,8.78,9.57(3 \times \mathrm{s}$, $3 \mathrm{H}, 3 \mathrm{NH}) .-\mathrm{C}_{14} \mathrm{H}_{22} \mathrm{~N}_{4} \mathrm{O}_{2}$ (278.3): calcd. C 60.40, H 7.96, N 20.12; found C 60.25, H 7.88, N 20.08.

Compound $2 e$ : M.p. $165-167{ }^{\circ} \mathrm{C}$. - Yield $84 \%$. - IR (KBr): $v=3306,3116,2938,1446,1695 \mathrm{~cm}^{-1} .-{ }^{1} \mathrm{H}$ NMR (300 MHz, [D $\left.\left.\mathrm{D}_{6}\right] \mathrm{DMSO}\right): \delta=3.48\left(\mathrm{~s}, 2 \mathrm{H} \mathrm{CH}_{2}\right), 3.55(\mathrm{~s}$, $\left.3 \mathrm{H}, \mathrm{NCH}_{3}\right), 5.82-7.01$ (m, 3H, Ar-H), 7.24-7.48 (m, 5H, Ar-H), 8.03, 8.93, $9.78(3 \times \mathrm{s}, 3 \mathrm{H}, 3 \mathrm{NH}) .-\mathrm{C}_{14} \mathrm{H}_{16} \mathrm{~N}_{4} \mathrm{O}_{2}$ (272.3): calcd. C 61.75, H 5.92, N 20.57; found C 61.82, H 5.89, N 20.63.

Compound 2f: M.p. $165-166{ }^{\circ} \mathrm{C}$. - Yield $78 \%$. - IR (KBr): $v=3300,3004,2941,1441,1684 \mathrm{~cm}^{-1} .-{ }^{1} \mathrm{H}$ NMR (300 MHz, [D 6 ]DMSO): $\delta=0.99$ (t, $J=7.1 \mathrm{~Hz}, 3 \mathrm{H}, \mathrm{CH}_{3}$ ), 3.03 (q, $\left.J=5.8 \mathrm{~Hz}, 2 \mathrm{H}, \mathrm{CH}_{2}\right), 3.35\left(\mathrm{~s}, 2 \mathrm{H}, \mathrm{CH}_{2}\right), 3.45$ (s, $3 \mathrm{H}$, $\left.\mathrm{NCH}_{3}\right), 5.79-6.61(\mathrm{~m}, 3 \mathrm{H}, \mathrm{Ar}-\mathrm{H}), 8.07,9.05,9.70(3 \times \mathrm{s}$, $3 \mathrm{H}, 3 \mathrm{NH}$ ). $-\mathrm{C}_{10} \mathrm{H}_{16} \mathrm{~N}_{4} \mathrm{O}_{2}$ (224.2): calcd. C 53.55, H 7.19, N 24.98; found C 53.48, H 7.28, N 25.01.

Preparation of 3-[(1-methyl-pyrrol-2-yl)methyl]-4-substituted-4,5-dihydro-1H-1,2,4-triazol-5-ones 3

A mixture of semicarbazide $2(10 \mathrm{mmol})$ and $40-50 \mathrm{~mL}$ of a $2 \%$ (in the case of compound $\mathbf{2 b} 10 \%$ ) aqueous solution of sodium hydroxide was boiled for $15-20 \mathrm{~h}(2 \mathrm{~h}$ for 2b). After cooling, the solution was neutralized with dilute hydrochloric acid. The precipitate was filtered off and then crystallized from ethanol. 
Compound 3a: M.p. $180-181{ }^{\circ}$ C. - Yield $89 \%$. - IR $(\mathrm{KBr}): v=3065,2948,1492,1704,1572,1492 \mathrm{~cm}^{-1}$. ${ }^{1} \mathrm{H}$ NMR (300 MHz, [D 6 ]DMSO): $\delta=3.38\left(\mathrm{~s}, 3 \mathrm{H}, \mathrm{NCH}_{3}\right)$, 3.79 (s, 2H, $\left.\mathrm{CH}_{2}\right), 5.54-6.56(\mathrm{~m}, 3 \mathrm{H}, \mathrm{Ar}-\mathrm{H}), 7.25-7.69$ (m, 5H, Ar-H), 11.60 (s, 1H, NH). $-\mathrm{C}_{14} \mathrm{H}_{13} \mathrm{~N}_{4} \mathrm{OBr}$ (333.2): calcd. C 50.46, H 3.93, N 16.81; found C 50.58, H 3.78, N 16.77 .

Compound 3b: M.p. $185-187{ }^{\circ} \mathrm{C}$. - Yield $80 \%$. - IR $(\mathrm{KBr}): v=3084,2938,1448,1709,1572,1493 \mathrm{~cm}^{-1}$. ${ }^{1} \mathrm{H}$ NMR (300 MHz, [D 6 ]DMSO): $\delta=3.44\left(\mathrm{~s}, 3 \mathrm{H}, \mathrm{NCH}_{3}\right)$, $3.75\left(\mathrm{~s}, 2 \mathrm{H}, \mathrm{CH}_{2}\right), 4.75$ (s, 2H, $\left.\mathrm{CH}_{2}\right), 5.74-6.63(\mathrm{~m}, 3 \mathrm{H}$, Ar-H), $7.13-7.34(\mathrm{~m}, 5 \mathrm{H}, \mathrm{Ar}-\mathrm{H}), 11.62(\mathrm{~s}, 1 \mathrm{H}, \mathrm{NH})$. ${ }^{13} \mathrm{C}$ NMR (300 MHz, [D $]$ DMSO): $\delta=23.63\left(\mathrm{CH}_{2}\right), 33.30$ $\left(\mathrm{CH}_{3}\right), 43.16\left(\mathrm{CH}_{2}\right), 106.26,107.80,122.41,124.77 ; 126.82$ 127.47, 128.62, $136.58($ all Ar-CH), $145.11(\mathrm{C}=\mathrm{N}), 155.17$ $(\mathrm{C}=\mathrm{O}) .-\mathrm{MS}(\mathrm{EI}, 70 \mathrm{eV}): \mathrm{m} / \mathrm{z}(\%)=268(35)\left[\mathrm{M}^{+}\right]$, 91 (100). $-\mathrm{C}_{15} \mathrm{H}_{16} \mathrm{~N}_{4} \mathrm{O}$ (268.3): calcd. C 67.14, H 6.01, N 20.88; found C 67.21, H 5.98, N 20.66.

Compound 3c: M. p. $183-184{ }^{\circ} \mathrm{C}$. - Yield $76 \%$. - IR $(\mathrm{KBr}): v=3079,2982,1459,1706,1577,1513 \mathrm{~cm}^{-1}$. ${ }^{1} \mathrm{H}$ NMR (300 MHz, [D 6 DMSO): $\delta=1.34(\mathrm{t}, J=6.9 \mathrm{~Hz}$, $\left.3 \mathrm{H}, \mathrm{CH}_{2}\right), 3.36\left(\mathrm{~s}, 3 \mathrm{H}, \mathrm{NCH}_{3}\right), 3.71\left(\mathrm{~s}, 2 \mathrm{H}, \mathrm{CH}_{2}\right), 4.06(\mathrm{q}$, $\left.J=7.0 \mathrm{~Hz}, 2 \mathrm{H}, \mathrm{CH}_{2}\right), 5.46-6.56(\mathrm{~m}, 3 \mathrm{H}, \mathrm{Ar}-\mathrm{H}), 6.96-7.18$ (m, $4 \mathrm{H}, \mathrm{Ar}-\mathrm{H}), 11.62$ (s, $1 \mathrm{H}, \mathrm{NH}) .-\mathrm{C}_{16} \mathrm{H}_{18} \mathrm{~N}_{4} \mathrm{O}_{2}$ (298.3): calcd. C 64.41, H 6.08, N 18.77; found C 64.68, H 6.28, N 18.59 .

Compound 3d: M.p. $218-219{ }^{\circ}$ C. - Yield $89 \%$. - IR $(\mathrm{KBr}): v=3068,2942,1451,1704,1574,1514 \mathrm{~cm}^{-1}$. ${ }^{1} \mathrm{H}$ NMR $\left(300 \mathrm{MHz},\left[\mathrm{D}_{6}\right] \mathrm{DMSO}\right): \delta=1.06-2.06(\mathrm{~m}, 10 \mathrm{H}$, $\left.5 \mathrm{CH}_{2}\right), 3.50\left(\mathrm{~s}, 3 \mathrm{H}, \mathrm{NCH}_{3}\right), 3.60-3.70(\mathrm{~m}, 1 \mathrm{H}, \mathrm{CH}), 3.91(\mathrm{~s}$, $\left.2 \mathrm{H}, \mathrm{CH}_{2}\right), 5.82-6.66(\mathrm{~m}, 3 \mathrm{H}, \mathrm{Ar}-\mathrm{H}), 11.34$ (s, 1H, NH). $\mathrm{C}_{14} \mathrm{H}_{20} \mathrm{~N}_{4} \mathrm{O}$ (260.3): calcd. C 64.58, H 7.74, N 21.52; found C 64.71, H 7.49, N 21.62.

Compound 3e: M.p. $157-158{ }^{\circ} \mathrm{C}$. - Yield $80 \%$. - IR $(\mathrm{KBr}): v=3078,2921,1456,1705,1579,1500 \mathrm{~cm}^{-1}$. ${ }^{1} \mathrm{H}$ NMR (300 MHz, [D 6 ]DMSO): $\delta=3.36$ (s, 3H, $\mathrm{NCH}_{3}$ ), $4.76\left(\mathrm{~s}, 2 \mathrm{H}, \mathrm{CH}_{2}\right), 5.41-6.55(\mathrm{~m}, 3 \mathrm{H}, \mathrm{Ar}-\mathrm{H}), 7.26-7.48$ $(\mathrm{m}, 5 \mathrm{H}, \mathrm{Ar}-\mathrm{H}), 11.73(\mathrm{~s}, 1 \mathrm{H}, \mathrm{NH}) .-{ }^{13} \mathrm{C} \mathrm{NMR}(300 \mathrm{MHz}$, [D $\mathrm{D}_{6}$ ]DMSO): $\delta=23.57\left(\mathrm{CH}_{2}\right), 33.16\left(\mathrm{CH}_{3}\right), 106.18,107.46$, 121.94, 125.04, 127.41, 128.50, 129.17, 132.83 (all Ar-CH), $144.99(\mathrm{C}=\mathrm{N}), 154.32(\mathrm{C}=\mathrm{O}) .-\mathrm{MS}(\mathrm{EI}, 70 \mathrm{eV}): \mathrm{m} / \mathrm{z}(\%)$ $=254(59)\left[\mathrm{M}^{+}\right], 94(100) .-\mathrm{C}_{14} \mathrm{H}_{14} \mathrm{~N}_{4} \mathrm{O}(254.3)$ : calcd . C 66.13, H 5.54, N 22.03: found C 66.32, H 5.68, N 21.91.

Compound 3f: M.p. $150-151{ }^{\circ} \mathrm{C}$. - Yield $90 \%$. - IR $(\mathrm{KBr}): v=3070,2930,1470,1703,1572,1520 \mathrm{~cm}^{-1}$. ${ }^{1} \mathrm{H}$ NMR (300 MHz, [D 6 DMSO): $\delta=0.97(\mathrm{t}, J=7.1 \mathrm{~Hz}$, $\left.3 \mathrm{H}, \mathrm{CH}_{3}\right), 3.51\left(\mathrm{q}, J=5.3 \mathrm{~Hz}, 2 \mathrm{H}, \mathrm{CH}_{2}\right), 3.74\left(\mathrm{~s}, 3 \mathrm{H}, \mathrm{NCH}_{3}\right)$, $3.91\left(\mathrm{~s}, 2 \mathrm{H}, \mathrm{CH}_{2}\right), 6.83-6.67(\mathrm{~m}, 3 \mathrm{H}, \mathrm{Ar}-\mathrm{H}), 11.47(\mathrm{~s}, 1 \mathrm{H}$, $\mathrm{NH}) .-{ }^{13} \mathrm{C}$ NMR $\left(300 \mathrm{MHz},\left[\mathrm{D}_{6}\right] \mathrm{DMSO}\right): \delta=13.86\left(\mathrm{CH}_{3}\right)$, $23.55\left(\mathrm{CH}_{2}\right), 33.38\left(\mathrm{CH}_{3}\right), 35.27\left(\mathrm{CH}_{2}\right), 106.29,107.74$, 122.37, $125.36($ all Ar-CH), $144.94(\mathrm{C}=\mathrm{N}), 154.87(\mathrm{C}=\mathrm{O})$. MS (EI, $70 \mathrm{eV}): \mathrm{m} / z(\%)=206(43)\left[\mathrm{M}^{+}\right], 108(100)$. $\mathrm{C}_{10} \mathrm{H}_{14} \mathrm{~N}_{4} \mathrm{O}$ (206.2): calcd. C 58.23, H 6.84, N 27.16; found C 58.47, H 6.95, N 27.01.

\section{Chromatographic analysis of lipophilicity}

Thin-layer chromatography was performed on $10 \times 10 \mathrm{~cm}$ TLC plates (Merck, Darmstadt, Germany) precoated with RP-18 $F_{245}$ gel. Methanol-water or acetonitrile-water mixtures were used as mobile phases. The methanol or acetonitrile content was varied from 50 to $70 \%(\mathrm{v} / \mathrm{v})$ in $5 \%$ steps. Plates were developed to a distance of $9 \mathrm{~cm}$ at r. t. in a horizontal DS chamber (Chromdes, Lublin, Poland) and after drying visualized under $\lambda=254 \mathrm{~nm}$ UV light. Unfortunately there was no possibility to visualize the solute 3d (lack of spot under UV light). Each experiment was performed in quadruplicate, coefficients of variation $(\mathrm{CV})$ of experimentally obtained $R_{\mathrm{F}}$ values were below $6 \%$. $R_{\mathrm{F}}$ values were used to calculate $R_{\mathrm{M}}$ by use of Eq. 2 .

$$
R_{\mathrm{M}}=\log \left(\frac{1}{R_{\mathrm{F}}}-1\right)
$$

The partition coefficients were calculated for the compounds by use of different theoretical procedures: $\log P_{\text {KOwIN }}$, Pallas Annlog $P$, Pallas Atomic 6, Pallas CDI-ReKKer, Pallas COMBIned, CHEM 3D $\log P$, CHEM 3D Partition Coefficient (octanol/water), $\log P$, SCILog $P$ Application Version 3.0, TITAN $\log P$ (Ghose-Crippen), HYPECHEM, MOLINSPIRATION lipophilicity (coefficients were determined by internet website [http://www.molinspiration. com/cgi-bin/properties?textMode=1]).

\section{Antimicrobial activity}

The newly synthesized compounds were screened for their in vitro activity against 9 reference species of aerobic bacteria (5 Gram-positive: Staphylococcus epidermidis ATCC 12228, Staphylococccus aureus ATCC 25923, Bacillus subtilis ATCC 6633, Bacillus cereus ATCC 10876, Micrococcus luteus ATCC 10240 and 4 Gram-negative: Escherichia coli ATCC 25922, Klebsiella pneumoniae ATCC 13883, Proteus mirabilis ATCC 12453, Pseudomonas aeruginosa ATCC 9027), and 4 reference species of fungi (Candida albicans ATCC 10231, Candida albicans ATCC 2091, Candida parapsilosis ATCC 22019, Aspergillus niger ATCC 16404). The inoculum density was adjusted to $0.5 \mathrm{McFar}-$ land standard with sterile saline $(0.85 \% \mathrm{NaCl})$, and then the suspensions were diluted $1: 10$ (for the agar well diffusion method) or $1: 100$ (for the broth dilution method) in MuellerHinton broth without (for bacteria) or with $2 \%$ glucose (for fungi). All stock solutions of the assayed compounds were prepared in $100 \%$ dimethylsulfoxide (DMSO). Using the agar well diffusion method, microbial suspensions were put onto Mueller-Hinton agar without (for bacteria) or with $2 \%$ glucose buffered at $\mathrm{pH}=5.6$ (for fungi). Then, a well was prepared in the plates with the help of a cork-borer $(0.85 \mathrm{~cm})$ in the agar medium, and $80 \mu \mathrm{L}$ of the tested compounds in concentrations of $1000-5000 \mathrm{mg} \mathrm{L}^{-1}$ were added to the wells. The same volumes of sterile $0.85 \% \mathrm{NaCl}$ or DMSO 
were used as a control. The plates were incubated at $37{ }^{\circ} \mathrm{C}$ for $18 \mathrm{~h}$ for bacteria and at $30{ }^{\circ} \mathrm{C}$ for $48 \mathrm{~h}$ for Candida spp. or $5 \mathrm{~d}$ for Aspergillus niger, depending on the compoundfree growth control. After incubation microbial growth was determined by measuring the diameter of zones of growth inhibition. The antibacterial assay for the compounds, which had shown promising activity against bacterias assessed by the first method, were also tested spectrophotometrically us- ing the broth dilution method. After incubation $\left(37^{\circ} \mathrm{C}\right.$ for $18 \mathrm{~h})$ optical density $\left(\mathrm{OD}_{600}\right)$ measurements were carried out for bacterial cultures in broth medium containing 31.25 to $500 \mathrm{mg} \mathrm{L}^{-1}$ of the compounds. The MIC (Minimal Inhibitory Concentration) values, defined as the lowest concentration of compound at which there is no visible growth of the tested bacteria, were determined by comparison with the growth of the control (compound-free) medium.
[1] J. L. Martinez, F. Baquero, Clin. Microbiol. Rev. 2002, 15, 647-679.

[2] H. Kitano, Prog. Drug Res. 2007, 64, 241 - 263.

[3] F. Clemense, C. Joliveau-Maushart, J. Meier, J. Creda, F. Delevallèe, J. Benzoni, R. Deraedt, Eur. J. Med. Chem. Chim. Ther. 1985, 20, 257-266.

[4] A. A. Ikizler, C. B. Johansson, O. Bekircan, C. Çelik, Acta Pol. Pharm.-Drug. Res. 1999, 56, 283 - 288.

[5] N. Demirbas, S. A. Karaoglu, A. Demirbas, K. Sancak, Eur. J. Med. Chem. 2004, 39, 793 - 804.

[6] A. Ikizler, F. Gümüs, U. Abbasoğlu, Pharmazie 1989, 44, 505-508.

[7] H. Yüksek, A. Demirbaş, A. Ikizler, C. B. Johansson, C. Çelik, A. A. Ikizler, Arzneim.-Forsch. 1997, 47, $405-409$.

[8] C.R. Katica, D. Vesna, K. Vlado, G. M. Dora, B. Alekrandra, Molecules 2001, 6, 815-824.

[9] R. J. Brown, D. A. Frasier, C. Happeresett, P. P. Castro, C. G. Sternberg, P. CT Int. Appl. WO 96,26,191, 1996.

[10] T. Murai, K. Kuriyama, Y. Hyama, R. Nagatahria, K. Ito, Jpn. Kokai Tokkyo Koho JP 0215, 069, 1990.

[11] S. Papakonstantinou-Garoufalias, E. Tani, O. Todoulou, A. Papadaki-Valiraki, E. Philippatos, E. De Clercq, J. Pharm. Pharmacol. 1998, 50, $117-124$

[12] Y. A. Al-Soud, M. N. Al-Dweri, N. A. Al-Masoudi, Il Farmaco 2004, 59, 775-783.

[13] J. Kane, B. Baron, M. Dudlej, S. Sorensen, M. Staeger, F. Miller, J. Med. Chem. 1990, 33, $2772-2777$.

[14] N. Demirbas, R. Ugurluoglu, A. Demirbas, Bioorg. Med. Chem. 2002, 10, 3717-3723.

[15] A. A. Ikizler, A. Ikizler, M. Serdar, N. Yildirim, Acta Pol. Pharm.-Drug. Res. 1997, 54, 363 - 370.

[16] S. Sun, H. Lou, Y. Gao, P. Fan, B. Ma, W. Ge, X. Wang, J. Pharm. Biomed. Anal. 2004, 34, 1117-1124.

[17] G. Verreck, K. Six, G. van der Mooter, L. Baert, J. P. Brewster, Int. J. Pharm. 2003, 251, 165 - 174.

[18] M. Clemons, R. E. Coleman, S. Verma, Cancer Treat. Rev. 2004, 30, 325 - 332.

[19] G. L. Biagi, M. Recanatini, A. M. Barbaro, P. A. Borea, Process Control and Quality 1997, 10, 129-149.

[20] V. Pliŝka, In Lipophilicity in Drug Action and Toxicology, VCV, Weinheim, 1996, pp. 263.

[21] K. Jóźwiak, H. Szumiło, E. Soczewiński, Wiad. Chem. 2001, 55, 1047 - 1074 (in Polish).
[22] T. Tuzimski, K. Sztanke, J. of Planar Chromatography 2005, 18, 274-281.

[23] A. Pyka, M. Babuśka, J. Śliwiok, J. of Planar Chromatography 2006, 19, $432-437$.

[24] J. Flieger, B. Modzelewska-Banachiewicz, H. Szumiło, M. Tatarczak, Chromatographia 2004, 60, S291-S294.

[25] G. Cimpan, M. Hadaruga, V. MIclaus, J. Chrom. A. 2000, $869,49-55$.

[26] T. Sławik, C. Kowalski, J. Chrom. A. 2002, 952, 295 299.

[27] J. Flieger, M. Tatarczak, M. Wujec, M. Pitucha, H. Szumiło, J. Planar Chromatogr. 2006, 19, $32-41$.

[28] J. Obniska, K. Kamiński, Biomed. Chromatogr. 2006, 20,1185-1191.

[29] M. Bajda, S. Boryczka, J. Wietrzyk, B. Malawska, Biomed. Chromatogr. 2007, 21, 123-131.

[30] D. Łażewska, P. Maludziński, E. Szymańska, K. KiećKononowicz, Biomed. Chromatogr. 2007, 21, $291-$ 298.

[31] Z. Mrkvićkova, P. Kovařikova, M. Doleżal, J. Planar Chromatogr. 2006, 19, $422-426$.

[32] J. Kresta, P. Kastner, V. Klimešová, J. Planar Chromatogr. 2005, 18, 450-454.

[33] D. Matosiuk, K. Jóźwiak, J. Planar Chromatogr. 2000, $13,52-56$.

[34] D. Matosiuk, K. Jóźwiak, J. Planar Chromatogr. 2000, $13,130-134$.

[35] A. Gumieniczek, A. Berecka, D. Matosiuk, H. Hopkała, J. Planar Chromatogr. 2007, 20, $261-265$.

[36] H. Kubinyi, QSAR: Hansch analysis and related approaches, VCH, Weinheim, 1993.

[37] A. Pyka, J. Planar Chromatogr. 2004, 17, 275-279.

[38] E. Brzezińska, G. Kośka, K. Walczyński, J. Chrom. A. 2003, 1007, $157-164$.

[39] J. Matysiak, A. Niewiadomy, G. Mącik-Niewiadomy, T. Korniłowicz, Eur. J. Med. Chem. 2000, 35, 393404.

[40] K. Więckowski, A. Czaja, A. Woźniak, A. Musiał, B. Malawska, J. Planar Chromatogr. 2007, 20, $101-$ 106.

[41] E. Soczewiński, C. A. Wachtmeister, J. Chromatogr. 1962, 7, 311-320.

[42] M. Pitucha, M. Wujec, M. Dobosz, Annales UMCS, Sectio AA, Lublin 2004, 59, 144-152. 